\title{
Causas de óbitos em hospital de ensino da Amazônia Ocidental brasileira
}

Causes of death in a teaching hospital of the Brazilian western Amazon Causas de fallecimiento en hospital universitario de la Amazonía Occidental brasileña

\author{
André Ricardo Maia da Costa de Faro' 1 (i) nttps:/orcid.org/0000-0003-0761-6070 \\ Andréia Moreira de Andrade ${ }^{1}$ ic https://orcid.org/0000-0003-2400-3794 \\ Fernanda Paula de Faria Guimarães ${ }^{2}$ io nttps://orcid.orgy/0000-0001-9142-1625 \\ Angélica Gonçalves Silva Belasco ${ }^{3}$ ib hittps://orcid.org/0000-0002-0307-6225 \\ Joao Luiz Grandi i io https://orcid.org/0000-0002-6522-7754 \\ Dulce Aparecida Barbosa ${ }^{3}$ io https://orcid.org/0000-0002-9912-4446
}

Como citar:

Faro AR, Andrade AM, Guimarães FP, Belasco AG, Grandi JL, Barbosa DA. Causas de óbitos em hospital de ensino da Amazônia Ocidental brasileira. Acta Paul Enferm. 2021;34:AAPE002515.

DOI

http://dx.doi.org/10.37689/actaape/2021A0002515

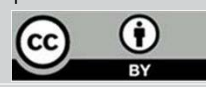

Mortalidade hospitalar; Epidemiologia; Sistemas de informação

Keywords

Hospital mortality; Cause of death; Epidemiology; Information system

Descriptores

Mortalidad hospitalaria; Causas de muerte Epidemiología; Sistemas de información

Submetido

8 de Setembro de 2020

Aceito

3 de Dezembro de 2020

Autor correspondente

André Ricardo Maia da Costa de Faro E-mail: enfermeiromaia@hotmail.com

\section{Resumo}

Objetivo: Identificar o perfil epidemiológico dos pacientes que evoluíram para óbito, caracterizar essas mortes segundo variáveis sociodemográficas e clínicas e analisar a relação entre a causa básica e a causa imediata do óbito em um hospital de ensino.

Métodos: Estudo quantitativo, transversal retrospectivo, com componentes descritivos, utilizando como principal fonte de dados os atestados de óbitos do Sistema de Informações do Sistema Único de Saúde e das declarações de óbitos ocorridos em 2016 da instituição pesquisada.

Resultados: Dos 634 óbitos, a maioria dos pacientes era do sexo masculino (56,9\%), sendo $70 \%$ de afrodescendentes, observou-se que quase metade $(48,8 \%)$ tinha 65 anos ou mais de idade, acometeu $50,7 \%$ dos homens acima dos 66 anos e $53,8 \%$ das mulheres com menos de 66 anos. As principais causas imediatas de morte foram associadas às neoplasias (45,4\%) e às doenças infecciosas e parasitárias $(28,9 \%)$. Quanto as declarações de óbitos, existe uma subnotificação nas causas consequenciais 2 e 3, de 12,6\% e $49,9 \%$, respectivamente.

Conclusão: A ocorrência de doenças infecciosas e parasitárias, os sinais/sintomas e exames clínicos/ laboratoriais alterados, exerceram impacto importante como causa imediata dos óbitos analisados. Destacase que os óbitos por neoplasias se mantiveram constantes em todas as categorias (causa imediata, causa consequencial 2, causa consequência 3, causa básica).

\section{Abstract}

Objectives: To identify the epidemiological profile of patients who died, to characterize these deaths according to sociodemographic and clinical variables and to analyze the relation between the underlying cause and immediate cause of death in a teaching hospital.

Methods: Quantitative cross-sectional study, with descriptive components, using as the main source of data the death certificates of the Information System of the Brazilian Unified Health System and the death declarations that occurred in 2016 in the institution studied.

Results: Of the 634 deaths, most of the patients were male (56.9\%), being 70\% Afro-descendants; it was observed that almost half (48.8\%) were 65 years of age or older; it affected $50.7 \%$ of men over 66 years and $53.8 \%$ of women under 66 years. The main immediate causes of death were associated with neoplasms (45.4\%) and infectious and parasitic diseases (28.9\%). Regarding death declarations, there is an underreporting in consequential causes 2 and 3 , of $12.6 \%$ and $49.9 \%$, respectively. 
Conclusion: The occurrence of infectious and parasitic diseases, the signs/symptoms and altered clinical/laboratory tests had an important impact as immediate cause of the analyzed deaths. It is noteworthy that deaths from neoplasms remained constant in all categories (immediate cause, consequential cause 2 , consequential cause 3 , and underlying cause).

\section{Resumen}

Objetivo: Identificar el perfil epidemiológico de los pacientes que fallecieron, caracterizar esas muertes de acuerdo con variables sociodemográficas y clínicas y analizar la relación entre la causa básica y la causa directa del fallecimiento en un hospital universitario.

Métodos: Estudio cuantitativo, transversal retrospectivo, con componentes descriptivos, utilizando como principal fuente de datos los certificados de defunción del Sistema de Información del Sistema Único de Salud y las declaraciones de las defunciones ocurridas en 2016 en la institución estudiada.

Resultados: De los 634 fallecimientos, la mayoría de los pacientes era de sexo masculino (56,9\%), el 70 \% era afrodescendiente. Se observó que casi la mitad (48,8 \%) tenía 65 años o más, el 50,7 \% de los hombres tenía más de 66 años y el 53,8 \% de las mujeres, menos de 66 años. Las principales causas directas de muerte se asociaron con neoplasias (45,4\%) y enfermedades infecciosas y parasitarias (28,9 \%). Respecto a las declaraciones de defunción, existe una subnotificación en la causa antecedente 2 del $12,6 \%$ y en la 3 del 49,9\%.

Conclusión: Los casos de enfermedades infecciosas y parasitarias, los signos/síntomas y estudios clínicos/de laboratorio alterados ejercieron un importante impacto como causa directa de los fallecimientos analizados. Se observa que los fallecimientos por neoplasias se mantuvieron constantes en todas las categorías (causa directa, causa antecedente 2 , causa antecedente 3 , causa básica).

\section{Introdução}

Os hospitais surgiram pautados no assistencialismo e dirigidos por religiosos e, na Idade Média, serviam apenas para segregar os doentes para evitar a propagação das doenças. Com a transformação dos hospitais como instrumento terapêutico no final do século XVIII, surge o profissional médico, com poderes sobre a vida e a morte humana, deixando de lado a autoridade sobre a vida e o morrer dos religiosos que dirigiam os hospitais da época. ${ }^{(1)}$

Somente no século $\mathrm{XX}$, que se instalam nos hospitais os modelos de medicalização, que serviriam não somente para a cura, mas também, para manter a disciplina hospitalar. ${ }^{(2)}$ Assim, os hospitais marcados pela complexidade da assistência, foram destinados para prover o cuidado com a saúde da sociedade desde o nascimento, a enfermidade até o momento da morte e, náo mais um local apenas para acolher as pessoas que iriam morrer. É neste contexto que a figura do médico passa a ser presença obrigatória, para estabelecer as regras das práticas hospitalares. ${ }^{(3)}$

Contudo é no final da Segunda Grande Guerra, que os avanços tecnológicos surgem para a cura e o prolongamento da vida, com a invenção dos ventiladores mecânicos, das máquinas para diálise e das unidades de terapia intensiva. ${ }^{(1)}$ Mas é nas últimas décadas que se observa um incremento das tecnologias voltadas à assistência em saúde, proporcionando maior acesso a novas terapias e métodos diagnósticos melhorando as condições das pessoas e a qualidade dos serviços, mas em contrapartida, aumentando os riscos de eventos adversos graves, tendo como consequências as mortes evitáveis em ambiente hospitalar. $^{(4)}$

No Brasil, os hospitais públicos e privados registram a cada hora, seis mortes em decorrência de eventos adversos graves, destas estima-se que quatro seriam mortes evitáveis. ${ }^{(5)}$ Entre as principais causas de mortes evitáveis, estariam os óbitos relacionados aos erros assistenciais, as infecçóes relacionadas à assistência à saúde (IRAS), entre elas as pneumonias, as infecçóes do sítio cirúrgico e as infecçôes urinárias, e aquelas provocadas pelos dispositivos vasculares, às complicações hemorrágicas e os erros no uso de medicamentos. ${ }^{(6)}$

Estudos reportam que as causas de mortes evitáveis em residentes no Brasil vêm caindo em 1,6\% ano e, as não evitáveis, em 1,4\% ao ano. Entretanto, os mesmo autores evidenciam um aumento de mortes para as causas específicas como as pneumonias $(1,9 \%$ ao ano) e as doenças não transmissíveis com redução de $2,2 \%$ ao ano, as quais foram mais elevadas nas faixas etárias de 60 a 69 anos de idade em 2013 (209.9/100.000hab). ${ }^{(7)}$ Contudo, é possível que ocorram mais mortes por eventos adversos do que é notificado, devido à qualidade das informações e da subnotificação dos casos. ${ }^{(6)}$

As mortes evitáveis ou redutíveis são todos os óbitos que podem ser prevenidos por açôes dos serviços de saúde. Número elevado de óbitos preveníveis pode estar associado a falhas no cuidado e são considerados eventos sentinela. ${ }^{(6)}$ As análises dos 
óbitos, das mortes evitáveis têm sido consideradas na literatura como um indicador de que a assistência integral não está funcionando bem. ${ }^{(7,8)}$

São poucos os estudos que se preocupam em avaliar as causas de óbitos nas diferentes faixas etárias, restringindo-se os dados a morte infantil ou a de causas específicas. ${ }^{(7)}$ Neste sentido, analisar os dados que possam identificar o número de óbitos em um hospital de referência e, os motivos que contribuíram para a ocorrência dos óbitos, pode ser uma das estratégias de se avaliar a eficácia do sistema de saúde da regiáo, permitindo auxiliar a autoridade sanitária local, para a criação de políticas de saúde pública. ${ }^{(8)}$

A enfermagem moderna, por sua vez, exerce um papel primoroso na condução das atividades assistenciais e tecnológicas das instituições de saúde e, consequentemente, dos hospitais, bem como no desempenho e funcionamento das unidades de internação, assumindo a responsabilidade pelos cuidados nas 24 horas do dia, durante os 7 dias da semana e ao longo dos 365 dias do ano, além de zelar pelo ambiente terapêutico e o suprimento dos materiais necessários. Em virtude de sua qualificação profissional, o protagonismo da enfermagem vai além da gestão da unidade de internação e, esta administração torna-se item fundamental que pode contribuir com a atuaçáo deste profissional no gerenciamento dos dados epidemiológicos (como um sistema de verificação de óbito, por exemplo) e até mesmo na manutenção da ordem nas diferentes tecnologias oferecidas na alta complexidade, desde que não sobrecarregue e não comprometa a atuação assistencial deste profissional.

Os objetivos deste estudo são identificar o perfil epidemiológico dos pacientes que evoluíram para óbito, caracterizar essas mortes segundo variáveis sociodemográficas e clínicas e analisar a relação entre a causa básica e a causa imediata do óbito em um hospital de ensino, localizado na capital da Amazônia ocidental brasileira.

\section{Métodos}

Desenho, período e local do estudo: Foi realizado estudo quantitativo, observacional, retrospectivo, utilizando como principal fonte de dados os atestados de óbitos, ocorridos entre janeiro e dezembro de 2016, registrados nos bancos nominais do Sistema de Informaçóes sobre Mortalidade (SIM) e Sistemas de Informações Hospitalares (SIH) do Sistema Único de Saúde (SUS), que contém informaçôes provenientes das cópias das declaraçôes de óbito do serviço de vigilância epidemiológica do hospital. Os dados coletados foram classificados de acordo com as variáveis sociodemográficas e as causas do óbito. O estudo foi realizado em um hospital público estadual, vinculado ao sistema de federal, do município de Rio Branco, no estado do Acre, localizado a sudeste da Região Norte e, considerado a capital da Amazônia ocidental brasileira. Exerce atividades nos níveis de atenção secundária e terciária, oferece serviços em regime ambulatorial e hospitalar e constitui-se como a principal unidade de atenção à média e alta complexidade de referência para o SUS em todo o Estado, como o Serviço de Atendimento Móvel de Urgência (SAMU), os hospitais e unidades mistas dos municípios acrianos, além de atender às demandas espontâneas do seu entorno como os municípios dos Estados do Amazonas e Rondônia e de países vizinhos como Peru e Bolívia.

Populaçáo e Amostra: A amostra do estudo foi obtida por todas as declaraçóes de óbito registradas no serviço de vigilância epidemiológica do hospital e inseridas no Sistema de Informaçóes sobre Mortalidade do SUS (SIM-SUS) de todos os pacientes que apresentavam registro de Internação no Sistema de Informaçóes Hospitalares do SUS (SIH-SUS). Os atestados de óbito foram agrupados e analisados conforme as causas, de acordo com a Classificação Estatística Internacional de Doenças e Problemas Relacionados com a Saúde - CID-10. ${ }^{(9)}$

Protocolo do Estudo: Os dados dos óbitos foram analisados utilizando-se a técnica da "linkage". Esta técnica baseia-se na ligação (unificação) de dois ou mais bancos de dados, obtidos a partir de informaçóes existentes entre os bancos, construindo a partir de um único sistema de informação, sem duplicidade, com maior fidelidade de informaçôes do mesmo indivíduo em diferentes sistemas e, posteriormente, no registro único gerado. Os instrumentos para coleta de dados foram codificados e valida- 
dos pelos pesquisadores, e, posteriormente, armazenados, em um único banco de dados, utilizando-se ferramenta Microsoft Excel, 2013, contendo as seguintes variáveis extraídas dos atestados de óbitos do Sistema de Informaçóes sobre Mortalidade do SUS: sexo, data de nascimento, etnia, situação conjugal, data, tempo e unidade de internação, data do óbito, procedência, causas imediatas e causas básicas dos óbitos. Considera-se "causa imediata" a doença ou estado mórbido que causou diretamente a morte. Considera-se "causa básica" a doença ou lesão que iniciou a sucessão de eventos mórbidos que levou diretamente à morte, e que motivou a internação. $\mathrm{O}$ controle de qualidade se deu por meio da dupla digitação utilizando-se digitadores diferentes, ao término da digitação procedeu-se a análise de consistência dos dados, onde se garantiu que os dados inconsistentes fossem conferidos e corrigidos na amostra, quando aplicável.

Como os registros das informaçôes do SIMSUS não apresentavam dados referentes a causa contribuinte do óbito, na categorização final os óbitos foram classificados em quatro grupos: 1) causa imediata; 2) causa consequencial 1 ; 2) causa consequencial 2 e, 3) causa básica, conforme descritos a seguir:

- Grupo 1: Causa imediata - Como causa imediata foram agrupadas as doenças classificadas nos Capítulos da CID-10, sendo: algumas doenças infecciosas e parasitárias (INF1), sinais, sintomas e achados anormais de exames clínicos e de laboratório, não classificados em outra parte (ANO1), doenças do aparelho respiratório (RES1), neoplasias (NEO1), doenças do aparelho circulatório (CIR1), doenças endócrinas, nutricionais e metabólicas (ENM1), doenças do aparelho digestivo (DIG1), doenças do aparelho geniturinário (GEN1) e outras (OUT1);

- Grupo 2: Causa consequencial 1 - Para este grupo foram definidas as doenças do aparelho respiratório (RES2), neoplasias (NEO2), algumas doenças infecciosas e parasitárias (INF2), doenças do aparelho geniturinário (GEN2), doenças do aparelho circulatório (CIR2), doenças do aparelho digestivo (DIG2), doenças en- dócrinas, nutricionais e metabólicas (ENM2), sinais, sintomas e achados anormais de exames clínicos e de laboratório, não classificados em outra parte (ANO2), doenças do sangue e dos órgáos hematopoiéticos e alguns transtornos imunitários (IMU2), Causas externas de morbidade e de mortalidade (EXT2) e outras (OUT2);

- Grupo 3: Causa consequencial 2 - Neste grupo foram definidas as seguintes doenças: neoplasias (NEO3), doenças do aparelho respiratório (RES3), doenças do aparelho digestivo (DIG3), doenças do aparelho circulatório (CIR3), doenças do aparelho geniturinário (GEN3), algumas doenças infecciosas e parasitárias (INF3), doenças endócrinas, nutricionais e metabólicas (ENM3) e outras (OUT3);

- Grupo 4: Causa básica - Neste grupo estão as neoplasias (NEO4), doenças do aparelho respiratório (RES4), doenças do aparelho circulatório (CIR4), algumas doenças infecciosas e parasitárias (INF4), doenças do aparelho digestivo (DIG4), doenças endócrinas, nutricionais e metabólicas (ENM4), doenças do aparelho geniturinário (GEN4), causas externas de morbidade e de mortalidade (EXT4) e outras (OUT4).

Análise dos Resultados e Estatística: A partir da planilha Excel, os dados foram exportados para o software IBM SPSS 14.0, adaptado para as variáveis selecionadas com a finalidade de analisar de forma descritiva e exploratória, com resultados apresentados em forma de tabelas. As variáveis categóricas foram descritas por frequência absoluta e frequência relativa. As variáveis numéricas por medidas de tendências centrais (médias e medianas) e medidas de dispersão (variância e desvio padrão).

Aspectos éticos: Depois de inserido na Plataforma Brasil, o estudo foi avaliado e aprovado sob o Certificado de Apresentação para Apreciação Ética (CAAE) No. 55573416.2.0000.5505, emitido pelo Comitê de Ética em Pesquisa da Universidade Federal de São Paulo (UNIFESP). A presente investigação atende os preceitos éticos baseados na normatização da Resolução 466/2012 do Conselho Nacional de Saúde. ${ }^{(10)}$ 
Foi assegurado o sigilo e confidencialidade das informaçóes de todos os dados coletados. Uma vez que se trata de dados secundários, coletados das declaraçôes de óbitos de pacientes já falecidos, dispensou-se o uso do Termo de Consentimento Livre e Esclarecido (TCLE), de acordo com parecer do CEP.

\section{Resultados}

Durante o ano de 2016, o município de Rio Branco registrou um total de 2.824 óbitos, destes $73,5 \%$ (2.076) ocorreram dentro de instituiçôes de saúde do município. $\mathrm{Na}$ instituição pesquisada, ocorreram 634 óbitos, o que corresponde a $22,5 \%$ do total de óbitos registrados no município, e uma prevalência de mortalidade neste hospital de 8,54\%. Os óbitos na instituição ocorreram em maior número no mês de abril, com 72 mortes (11,0\%) e, em menor, no mês de novembro, com 36 (5,7\%). A média de mensal foi de 52,8 óbitos.

$\mathrm{Na}$ tabela 1 , são analisadas as variáveis sociodemográficas dos pacientes que faleceram na instituição no ano de 2016. Em relação ao sexo, os óbitos ocorreram mais entre homens, com 361 (56,9\%), do que entre as mulheres com 273 (43,1\%). Quanto à idade, 50,7\% dos óbitos ocorreram entre homens acima dos 66 anos de idade enquanto que 53,8\% foram registrados entre mulheres com idade inferior a 66 anos.

Quanto à etnia registrada nos atestados de óbito, verifica-se que cerca de $70 \%$ eram de afrodescendentes, havendo uma distribuição normal entre homes e mulheres. Interessante ressaltar que dentre as outras etnias apenas seis óbitos $(0,9 \%)$ foram entre indígenas. Quando se analisa o estado conjugal dos falecidos, observa-se que cerca que $25,7 \%$ das mulheres são viúvas, contra $46,3 \%$ dos homens casados ou vivendo em união estável, no momento do óbito. Ao analisarmos separadamente, o registro de parceiro estável nas declarações de óbitos, verifica-se que $46,0 \%$ dos homens contra $60,1 \%$ das mulheres não tinham parceiro (a) estável quando faleceram.

Interessante ressaltar que cerca de $30 \%$ dos óbitos analisados ocorreram entre as mulheres em até três dias de internação, enquanto que para os homens, 52,3\% das ocorrências foram de onze a 23 dias ou mais de internação. (Tabela 1).

Os óbitos, em sua grande maioria, ocorreram nas unidades críticas, sendo que $24,9 \%$ dos homens e 20,8\% das mulheres vieram a falecer nas unidades de terapia intensiva, seguido dos óbitos na unidade de geriatria. As unidades que menos registraram, no período do estudo, ocorrências de óbitos foram as clinicas de nefrologia (hemodiálise e dialise) e a clínica cirúrgica com $0,6 \%$ e $2,1 \%$ do total de óbitos, respectivamente. (Tabela 1 ).

Tabela 1. Distribuição dos óbitos ocorridos em hospital de ensino de acordo com as variáveis sociodemográficas, o tempo de permanência e a unidade de ocorrência $(n=634)$

\begin{tabular}{|c|c|c|c|}
\hline Variáveis & $\begin{array}{c}\text { Masculino } \\
\mathrm{n}(\%)\end{array}$ & $\begin{array}{c}\text { Feminino } \\
\mathrm{n}(\%)\end{array}$ & $\begin{array}{l}\text { Total } \\
\mathrm{n}(\%)\end{array}$ \\
\hline \multicolumn{4}{|l|}{ Faixa Etária } \\
\hline Até 53 anos de idade & $84(23,3)$ & $78(28,6)$ & $162(25,5)$ \\
\hline De 54 a 65 anos de idade & $94(26,0)$ & $69(25,2)$ & $163(25,7)$ \\
\hline De 66 a 76 anos de idade & $103(28,5)$ & $63(23,1)$ & $166(26,2)$ \\
\hline De 76 anos de idade ou mais & $80(22,2)$ & $63(23,1)$ & $143(22,6)$ \\
\hline Total & $361(100,0)$ & $273(100,0)$ & $634(100,0)$ \\
\hline \multicolumn{4}{|l|}{ Etnia } \\
\hline Afrodescendentes & $261(67,7)$ & $203(20,7)$ & $464(68,9)$ \\
\hline Brancos & $84(23,3)$ & $58(21,3)$ & $142(22,4)$ \\
\hline Outras (oriental/indígena) & $4(1,0)$ & $3(1,1)$ & $7(0,2)$ \\
\hline Ignorada & $12(3,4)$ & $9(3,3)$ & $21(3,3)$ \\
\hline Total & $361(100,0)$ & $273(100,0)$ & $634(100,0)$ \\
\hline \multicolumn{4}{|l|}{ Situação conjugal } \\
\hline Solteiro & $115(31,9)$ & $81(29,7)$ & $196(30,9)$ \\
\hline Casado ou em união estável & $167(46,3)$ & $88(32,3)$ & $255(40,2)$ \\
\hline Viúvo & $31(8,6)$ & $70(25,7)$ & $101(16,0)$ \\
\hline Separado/divorciado & $20(5,5)$ & $13(4,7)$ & $33(5,2)$ \\
\hline Ignorado & $28(7,7)$ & $21(7,7)$ & $49(7,7)$ \\
\hline Total & $361(100,0)$ & $273(100,0)$ & $634(100,0)$ \\
\hline \multicolumn{4}{|l|}{ Tempo de internação (dias) } \\
\hline Até 3 & $92(25,5)$ & $84(30,8)$ & $176(27,8)$ \\
\hline De 4 a 10 & $80(22,2)$ & $66(24,2)$ & $146(23,0)$ \\
\hline De 11 a 23 & $94(26,0)$ & $63(23,0)$ & $157(24,8)$ \\
\hline 23 ou mais & $95(26,3)$ & $60(22,0)$ & $155(24,4)$ \\
\hline Total & $361(100,0)$ & $273(100,0)$ & $634(100,0)$ \\
\hline \multicolumn{4}{|l|}{ Unidade na constatação do óbito } \\
\hline Clínica oncológica & $47(13,1)$ & $43(15,8)$ & $90(14,2)$ \\
\hline Doenças infecciosas & $34(9,5)$ & $15(5,5)$ & $49(7,7)$ \\
\hline Clínica cirúrgica & $7(1,9)$ & $6(2,2)$ & $13(2,1)$ \\
\hline Clínica médica & $16(4,5)$ & $9(3,3)$ & $25(3,9)$ \\
\hline Geriatria & $78(21,6)$ & $72(26,3)$ & $150(23,7)$ \\
\hline Nefrologia/Hemodiálise & $2(0,5)$ & $2(0,7)$ & $4(0,6)$ \\
\hline Emergência Oncológica & $44(12,2)$ & $38(13,9)$ & $82(12,9)$ \\
\hline Unidade de Terapia Intensiva & $90(24,9)$ & $57(20,8)$ & $147(23,2)$ \\
\hline Terapia Semi-intensiva & $17(4,6)$ & $7(2,6)$ & $24(3,8)$ \\
\hline Sem Informação & $26(7,2)$ & $24(8,9)$ & $50(7,9)$ \\
\hline Total & $361(100,0)$ & $273(100,0)$ & $634(100,0)$ \\
\hline
\end{tabular}


$\mathrm{Na}$ tabela 2, são apresentadas as causas imediata e básica de morte de acordo com a classificaçáo dos Capítulos da CID-10.

Quando analisamos as causas imediatas dos óbitos, verifica-se que $183(28,9 \%)$ foram entre aquelas relacionadas no Capítulo 1 da CID-10, que trata de algumas doenças infecciosas e parasitárias, seguidas do capítulo XVIII que trata dos sintomas, sinais e achados anormais de exames clínicos e de laboratório, não classificados em outra parte, com 137 (21,6\%) dos óbitos. Contudo, quando analisamos a causa básica, verifica-se que 288 (15,4\%) foram óbitos em decorrência das neoplasias/tumores (Capítulo II da CID-10). Os subgrupos de doenças que não registraram nenhum óbito com causa imediata estão nos Capítulos XII - doenças da pele e do tecido conjuntivo (causas dermatológicas), e XIII que trata das doenças do sistema osteomuscular e do tecido conjuntivo (causas ortopédicas ou musculares). Já para a causa básica, o Capítulo XIX que trata das lesóes, envenenamentos e algumas outras consequências de causas externas, também, não houve registros de óbitos.

Tabela 2. Distribuição das causas imediata e básica dos óbitos classificados de acordo com os Capítulos da CID-10 informados nos atestados de óbito do SIM-SUS ( $n=634)$

\begin{tabular}{llcc}
\hline $\begin{array}{l}\text { Capítulos } \\
\text { CID-10 }\end{array}$ & Subgrupos das doenças e sistemas & $\begin{array}{c}\text { Imediata } \\
\mathbf{n}(\%)\end{array}$ & $\begin{array}{c}\text { Básica } \\
\mathbf{n}(\%)\end{array}$ \\
\hline I & Algumas doenças infecciosas e parasitárias & $183(28,9)$ & $45(7,1)$ \\
II & Neoplasmas (tumores) & $102(16,1)$ & $288(15,4)$ \\
III & Doenças do sangue dos órgãos hematopoiéticos & $5(0,8)$ & $4(0,6)$ \\
IV & Doenças endócrinas, nutricionais e metabólicas. & $20(3,2)$ & $35(5,5)$ \\
V & Transtornos e doenças do sistema nervoso & - & $2(0,3)$ \\
VI & Doenças do sistema nervoso & $3(0,5)$ & $5(0,8)$ \\
IX & Doenças do aparelho circulatório & $34(5,4)$ & $85(13,4)$ \\
X & Doenças do aparelho respiratório & $111(17,5)$ & $92(14,5)$ \\
XI & Doenças do aparelho digestivo & $17(2,7)$ & $36(5,7)$ \\
XII & Doenças da pele e do tecido subcutâneo & - & $6(0,9)$ \\
XIII & Doenças do sistema osteomuscular e conjuntivo & - & $4(0,6)$ \\
XIV & Doenças do aparelho geniturinário & $12(1,9)$ & $18(2,8)$ \\
XV & Gravidez, parto e puerpério. & $1(0,2)$ & $1(0,2)$ \\
XVI & Algumas afecções originadas no período neonatal & $2(0,3)$ & $1(0,2)$ \\
XVIII & Sintomas, sinais e achados anormais. & $137(21,6)$ & $2(0,3)$ \\
XIX & Lesões, envenenamentos e outras causas externas. & $4(0,6)$ & - \\
XX & Causas externas de morbidade e mortalidade & $3(0,5)$ & $10(1,6)$ \\
\hline
\end{tabular}

Na tabela 3, são demonstradas as distribuiçóes dos óbitos de acordo com as causas de ocorrência. Assim, a principal causa imediata de óbito foram as doenças infecciosas e parasitárias, com 183 (28,9\%) óbitos, seguidas dos sinais e sintomas anormais com 137 (21,6\%). Entretanto, quando analisamos as causas consequenciais, verifica-se que as doenças respiratórias foram responsáveis por 161 (25,4\%) do total de casos do Grupo 2 e as neoplasias com 13,6\% do total dos óbitos do Grupo 3. Porém, quando verificamos a causa básica, a taxa de mortalidade do hospital concentra-se nas neoplasias, com 45,4\% do total dos óbitos registrados na instituição. Chama atenção em nossa casuística, a frequência nas perdas relacionadas as causas consequenciais $2 \mathrm{e}$ 3 , de $12,6 \%$ e $49,9 \%$, respectivamente.

Tabela 3. Distribuição das causas dos óbitos de acordo com os Capítulos da CID-10, relacionadas com a causa imediata, as causas consequenciais e as causas básicas, ocorridos em um hospital de ensino $(n=634)$

\begin{tabular}{|c|c|c|c|c|}
\hline Capítulos CID-10 & $\begin{array}{c}\text { Causa } \\
\text { Imediata (1) } \\
n(\%)\end{array}$ & $\begin{array}{c}\text { Causa } \\
\text { Consequencial } \\
(2) \\
n(\%) \\
\end{array}$ & $\begin{array}{c}\text { Causa } \\
\text { Consequencial } \\
(3) \\
\mathrm{n}(\%) \\
\end{array}$ & $\begin{array}{c}\text { Causa } \\
\text { Básica (4) } \\
\text { n(\%) }\end{array}$ \\
\hline Infecciosas & $183(28,9)$ & $62(9,8)$ & $22(3,5)$ & $45(7,1)$ \\
\hline Anormais & $137 \quad(21,6)$ & $19(3,0)$ & - & - \\
\hline Respiratórias & $111(17,5)$ & $161(25,4)$ & $81(12,8)$ & $92(14,5)$ \\
\hline Neoplasias & $102(16,1)$ & $114(18,0)$ & $86(13,6)$ & $288(45,4)$ \\
\hline Circulatórias & $34(5,4)$ & $45(7,1)$ & $38(6,0)$ & $85(13,4)$ \\
\hline Endócrinas & $20(3,2)$ & $23(3,6)$ & $17(2,7)$ & $35(5,5)$ \\
\hline Digestivas & $17(2,7)$ & $37(5,8)$ & $40(6,3)$ & $36(5,7)$ \\
\hline Geniturinárias & $12(1,9)$ & $57(9,0)$ & $25(3,9)$ & $18(2,8)$ \\
\hline Imunológicas & - & $11(1,7)$ & - & - \\
\hline Externas & & $10(1,6)$ & - & $10(1,6)$ \\
\hline Outras causas & $18(2,8)$ & $15(2,4)$ & $15(2,4)$ & $25(3,9)$ \\
\hline Perdas & - & $80(12,6)$ & $310(49,9)$ & - \\
\hline Total & $634(100,0)$ & $554(87,4)$ & $324(51,1)$ & $634(100,0)$ \\
\hline
\end{tabular}

Na tabela 4, podemos observar a frequência relativa e absoluta das causas de óbito de acordo com os subgrupos da CID-10, no momento da internação hospitalar comparativamente ao desfecho (causa básica versus causa imediata do óbito).

As doenças infecciosas e parasitárias foram a principal causa imediata de óbitos com $183(28,9 \%)$ do total dos casos, seguida dos agravos do Capítulo XVIII da CID-10, que trata dos sintomas, sinais e achados anormais, com 137 óbitos $(21,6 \%)$ do total de casos.

Já para a causa consequencial dos óbitos as doenças do aparelho respiratório foram responsáveis por $161(25,4 \%)$ dos casos dos óbitos nesta categoria. Quando analisamos separadamente os óbitos decorrentes das neoplasias, verifica-se que 288 (45,4\%) 
Tabela 4. Distribuição das frequências absoluta e relativa da relação entre a Causa Imediata (1) e a Causa Básica (4) dos óbitos ocorridos em hospital de ensino $(n=634)$

\begin{tabular}{|c|c|c|c|c|c|c|c|c|c|c|}
\hline \multirow{3}{*}{ Causa 4} & \multicolumn{10}{|c|}{ Causa 1} \\
\hline & INF1 & AN01 & RES1 & NE01 & CIR1 & ENM1 & DIG1 & GEN1 & OU1 & Total \\
\hline & $\mathrm{n}(\%)$ & $\mathrm{n}(\%)$ & $\mathrm{n}(\%)$ & $\mathrm{n}(\%)$ & n(\%) & $\mathrm{n}(\%)$ & $\mathrm{n}(\%)$ & $\mathrm{n}(\%)$ & $\mathrm{n}(\%)$ & n(\%) \\
\hline NEO4 & $43(14,9)$ & $56(19,4)$ & $44(15,3)$ & $102(35,4)$ & $14(4,9)$ & $13(4,5)$ & $6(2,1)$ & $4(1,4)$ & $6(2,1)$ & $288(45,4)$ \\
\hline RES4 & $42(45,7)$ & $15(16,3)$ & $31(33,7)$ & - & $2(2,2)$ & $1(1,1)$ & - & $1(1,1)$ & - & $92(14,5)$ \\
\hline CIR4 & $30(35,3)$ & $24(28,2)$ & $16(18,8)$ & - & $10(11,8)$ & - & $1(1,2)$ & $2(2,4)$ & $2(2,4)$ & $85(13,4)$ \\
\hline INF4 & $20(44,4)$ & $14(31,1)$ & $6(13,3)$ & - & - & $1(1,2)$ & $3(6,7)$ & - & $1(1,2)$ & $45(7,1)$ \\
\hline DIG4 & $14(38,9)$ & $13(36,1)$ & $2(5,6)$ & - & $1(2,8)$ & $1(2,8)$ & $5(13,9)$ & - & - & $36(5,7)$ \\
\hline ENM4 & $12(34,3)$ & $4(11,4)$ & $7(20,0)$ & - & $3(8,6)$ & $3(8,6)$ & $1(2,9)$ & $4(11,4)$ & $1(2,9)$ & $35(5,5)$ \\
\hline GEN4 & $8(44,4)$ & $3(16,7)$ & $1(5,6)$ & - & $2(11,1)$ & $1(5,6)$ & $1(5,6)$ & - & $2(11,1)$ & $18(2,8)$ \\
\hline EXT4 & $2(20,0)$ & $1(10,0)$ & $2(20,0)$ & - & $1(10,0)$ & - & - & - & $4(40,0)$ & $10(1,6)$ \\
\hline OUT4 & $12(48,0)$ & $7(28,0)$ & $2(8,0)$ & - & $1(4,0)$ & - & - & $1(4,0)$ & $2(8,0)$ & $25(3,0)$ \\
\hline TOTAL & $183(28,9)$ & $137(21,6)$ & $111(17,5)$ & $102(16,1)$ & $34(5,4)$ & $20(3,2)$ & $17(2,7)$ & $12(1,9)$ & $18(2,8)$ & $634(100,0)$ \\
\hline
\end{tabular}

IINF - algumas doenças infecciosas e parasitárias; NEO - Neoplasmas (tumores); ENM - Doenças endócrinas, nutricionais e metabólicas; CIR - Doenças do aparelho circulatório; RES - Doenças do aparelho respiratório; DIG - Doenças do aparelho digestivo; GEN - Doenças do aparelho geniturinário; ANO - Sintomas, sinais e achados anormais de exames clínicos e de laboratório, não classificados em outra parte; EXT - Causas externas de morbidade e de mortalidade; OUT - outras

foram decorrentes de causa básica. Entretanto, não podemos deixar de observar que os óbitos por neoplasias se mantiveram constantes em todas as categorias, com média de apenas $15,9 \%$ para as demais causas: imediata e consequencial (Tabela 4), o que representa que $31,6 \%$ dos pacientes com tumores foram a óbito por outras causas decorrentes da doença e não propriamente da doença que motivou a internação.

\section{Discussão}

Com os dados apresentados neste estudo, foi possível realizar a análise dos óbitos em um hospital estadual, vinculado ao sistema de ensino federal, localizado na capital da Amazônia Ocidental brasileira, ocorridos durante o ano de 2016. A mortalidade hospitalar é um tradicional indicador de desempenho institucional e corresponde a uma ferramenta bastante útil nas condições em que a morte não é evento raro, para indicar serviços com eventuais problemas de qualidade. $^{(11,12)}$

Quanto ao sexo declarado nos atestados de óbito, verificou-se que a mortalidade foi maior entre os pacientes do sexo masculino do que entre o feminino, dados corroborados com outros estudos que também, referem mortalidade maior entre os homens. ${ }^{(13-15)}$

Em relação à faixa etária dos pacientes e acompanhando as estatísticas de envelhecimento da população brasileira, observou-se que quase metade da amostra deste estudo (48,8\%), evoluiu para óbito na instituiçáo com 65 ou mais anos de idade. Ressalta-se que, no Brasil, o número de indivíduos acima dos 60 anos de idade mais que dobrou nas últimas seis décadas. ${ }^{(16,17)}$

$\mathrm{Na}$ maioria dos países, o envelhecimento da população é uma das transformaçóes sociais mais significativas do século XXI em decorrência do progressivo declínio nas suas taxas de mortalidade e de fecundidade, refletindo na elevação da expectativa de vida com aumento da população em idades mais avançadas. ${ }^{(18,19)}$

Por se tratar de uma instituição que é considerada referência estadual na área oncológica, o que pode impactar na taxa de mortalidade do estudo, a principal causa dos óbitos deu-se em decorrência das neoplasias $(45,4 \%)$, contrapondo-se ao segundo lugar tanto dos países desenvolvidos, quanto daqueles em desenvolvimento. ${ }^{(8)}$

Observa-se também que o número de mortes em pacientes com doenças crônicas, principalmente, as dos aparelhos respiratório $(14,5 \%)$ e circulatório $(13,4 \%)$ e as doenças infecciosas e parasitárias $(7,1 \%)$ foi elevado. Tais dados são comparados com os encontrados em hospital de urgência localizado em Sergipe que aponta como principal causa de morte as doenças infecciosas e parasitárias e as causas externas. ${ }^{(8)}$ Contudo, tais dados não são corroborados com os encontrados no Rio Grande do Sul, que apontam índices de mortalidade para as doenças do aparelho circulatório de $32 \%$, para as neoplasias e tumores em torno de $25 \%$ e no grupo 
de algumas doenças infecciosas e parasitárias um índice de $4,7 \%$ do total geral das mortes ocorridas em 2013. ${ }^{(8)}$

Merece destaque o alto índice de mortes associadas às doenças infecciosas, onde $28,9 \%$ dos óbitos foram associados a este grupo de agravos como causa imediata do óbito, valor que se encontra acima da média mundial de $23 \%{ }^{(20)}$ Tais dados não são corroborados com os dados apresentados pelo Ministério da Saúde, que não evidenciam como as três principais causas de morte no Brasil, no ano de 2012, aquelas associadas às doenças infecciosas e parasitárias. ${ }^{(21)}$ Contudo, os dados se assemelham aos encontrados em outros dois estudos, sendo um conduzido no estado de Sergipe e outro em hospital realizado em hospital no Estado de São Paulo, onde $30,8 \%$ e $15,4 \%$ das mortes estiveram associadas à IRAS, respectivamente. ${ }^{(5,8)}$

Dados referentes ao encadeamento das doenças infecciosas e parasitárias que culminaram com a morte desta população, mostram que $7,1 \%$ estiveram relacionadas como a causa básica do óbito, 9,8\% e $3,5 \%$ como causa consequencial 2 e 3 , respectivamente, e 28,9\% como causa imediata do óbito. Esta representação demonstra que as doenças infecciosas entraram na cadeia de eventos responsáveis pela morte destes pacientes, deduzindo que este achado se originou durante a internação. Há que se destacar que as IRAS constituem uma relevante causa de morbidade com alta letalidade e, é considerada atualmente, um grave problema de saúde pública. ${ }^{(22-24)}$

Percebe-se que além da gravidade do quadro clínico e do aumento do tempo de internação, é possível destacar ainda os custos imensuráveis a que pacientes e familiares estão suscetíveis, além da dor e do sofrimento que afetam sobremaneira a qualidade de vida, podendo produzir sequelas físicas el ou psicológicas e até mesmo a morte relacionada a ocorrência de IRAS. ${ }^{(22)}$ É evidente que os hospitais de ensino são os mais vulneráveis à ocorrência deste tipo de agravo. ${ }^{(25)}$

Avaliando a relação entre causa imediata e causa básica dos óbitos, verificou-se que os pacientes acometidos por RES4, INF4 e GEN4 apresentaram INF1 como causa imediata em mais de $40 \%$ dos óbitos (RES4 45,7\%, INF4 44,4\% e GEN4 44,4\%).
Ressalta-se que nas doenças do aparelho respiratório é comum a utilização de dispositivos invasivos como a intubação orotraqueal e o uso de ventilaçáo mecânica. Da mesma forma, nas doenças do aparelho geniturinário também é rotineiro a utilização de cateter vesical de demora, como dispositivo invasivo. Em ambos os casos, já é conhecida a associação entre uso de dispositivos invasivos e a ocorrência de IRAS, pneumonias e infecções do trato urinário. ${ }^{(26-29)}$

Ao compararmos isoladamente as causas principais de óbito no ano de 2016 no estado do Acre e 2013 no Rio Grande do Sul, percebe-se uma ligeira mudança no padrão das doenças, sendo que para as mortes do sul as principais causas são as doenças típicas de países industrializadsos, as doenças cardiovasculares e as neoplasias com 32\% e $25 \%$ respectivamente, enquanto que no estado do Norte a inversão ocorre para as doenças típicas de país em desenvolvimento como as doenças infecciosas com $28,9 \%$ contra apenas $4,7 \%$ para este grupo no sul. (8) Interessante observar que em ambas as regióes brasileiras as doenças provocadas pela contaminação ambiental são muito parecidas, com 15,4\% das mortes principais ocorridas por doenças respiratórios no sul e $17,5 \%$ no norte. ${ }^{(8,13)}$

Por fim, observou-se que a busca ativa realizada pelos profissionais do SUS para o preenchimento das lacunas existentes nas declaraçóes de óbitos foram de extrema importância para garantir o preenchimento das causas imediata e básica dos óbitos, atendendo ao desejável indicador de perdas e inconsistências preconizado pelo Ministério da Saúde em menor que $10 \%{ }^{(29)}$

Dada a escassez de estudos regionais, e até mesmo nacionais, dessa magnitude, sugere-se mais investigaçóes, descritivas e analíticas, no sentido de conhecer com mais clareza não apenas os possíveis problemas que levaram a esse perfil de mortalidade, mas também as possíveis intervençóes, pensando em prevenção, melhoria da qualidade da assistência e do impacto da Atenção Primária na prevenção desses casos que chegaram inevitavelmente a óbito na alta complexidade. Esses resultados contribuirão em tomadas de decisões e na redução de danos causados aos pacientes que buscam assistência nos hospitais. 


\section{Conclusão}

Os óbitos ocorridos em hospital de ensino na Amazônia Ocidental brasileira a maioria era do sexo masculino $(56,9 \%)$, sendo $70 \%$ de afrodescendentes, onde observou-se que quase metade $(48,8 \%)$ tinha 65 anos ou mais de idade, acometeu 50,7\% dos homens acima dos 66 anos e 53,8\% das mulheres com menos de 66 anos, e $25,7 \%$ das mulheres viúvas contra $46,3 \%$ dos homens casados ou vivendo em união estável. As principais causas imediatas de morte foram associadas às neoplasias (45,4\%) e às doenças infecciosas e parasitárias (28,9\%). A principal causa imediata de óbito foram as doenças infecciosas e parasitárias, com 183 (28,9\%) óbitos, seguidas dos sinais e sintomas anormais com 137 $(21,6 \%)$. Destaca-se que os óbitos por neoplasias se mantiveram constantes em todas as categorias (causa imediata, causa consequencial 2, causa consequência 3, causa básica). Quanto as declaraçóes de óbitos, existe uma subnotificação nas causas consequenciais 2 e 3 , de $12,6 \%$ e $49,9 \%$, respectivamente.

\section{Agradecimentos}

Ao Instituto Federal do Acre e ao Conselho Nacional de Desenvolvimento Científico e Tecnológico pelo fomento de bolsa de doutorado por intermédio do Edital No 04/2016 PROINP/IFAC.

\section{Colaborações}

Faro ARMC, Andrade AM, Guimarães FPF, Belasco AGS, Grandi JL e Barbosa DA contribuíram com a concepção do estudo, análise e interpretação dos dados, redação do artigo, revisáo crítica relevante do conteúdo intelectual e aprovação da versão final a ser publicada.

\section{Referências}

1. Menezes RA. A medicalização da esperança: reflexões em torno de vida, saúde/doença e morte. Amazón. Rev Antropol. 2013;5(2):47898.
2. Silva DC, Rocha Júnior JR. 0 hospital é o lugar da saúde? A psicologia da saúde frente ao processo saúde-doença. Interfaces Científicas Saúde e Ambiente. 2015:4(1):9-17.

3. de Araújo KM, Leta J. Os hospitais universitários federais e suas missões institucionais no passado e no presente. Hist Cienc Saude Manguinhos. 2014;21(4):1261-81.

4. Guimarães AC, Donalisio MR, Santiago TH, Freire JB. Óbitos associados à infecção hospitalar, ocorridos em um hospital geral de Sumaré-SP, Brasil. Rev Bras Enferm. 2011;64(5):864-9.

5. Couto RC, Pedrosa TM, Roberto BA, Daibert PB, Abreu AC, Leao ML. II Anuário da Segurança Assistencial Hospitalar no Brasil. Propondo as prioridades nacionais. Belo Horizonte: Instituto de Estudos de Saúde Suplementar; 2018.

6. Malta DC, Saltarelli RM, Prado RR, Monteiro RA, Almeida MF. Mortes evitáveis no Sistema Único de Saúde na população brasileira, entre 5 e 69 anos, 2000 - 2013. Rev Bras Epidemiol. 2018;21:e180008.

7. Alves AT, Faro A. Perfil epidemiológico da mortalidade em um hospital de urgência em Sergipe. J Health Biol Sci. 2016;4(2):95-101.

8. Saltarelli RMF, Prado RRD, Monteiro RA, Machado ÍE, Teixeira BSM, Malta DC. Deaths preventable by actions of the Unified Health System in the population of the Brazilian Southeast Region. Cien Saude Colet. 2019;24(3):887-898.

9. Organizaçâo Mundial Da Saúde (OMS). Classificação Estatística Internacional de Doenças e Problemas relacionados à Saúde - $1^{\text {a }}$ Revisão. CID-10. São Paulo: EDUSP; 1997.

10. Brasil. Ministério da Saúde. Conselho Nacional de Saúde. Resolução No. 466 de 12 de dezembro de 2010. Brasília (DF): Ministério da Saúde; 2010. [citado 2019 Ago]. Disponível em: http://bvsms.saude. gov.br/bvs/saudelegis/cns/2013/res0466_12_12_2012.html

11. Gomes AS, Klück MM, Riboldi J, Fachel JM. Mortality prediction model using data from the Hospital Information System. Rev Saude Publica. $2010 ; 44(5): 934-41$.

12. Secretaria de Planejamento, Orçamento e Gestão. Coeficiente de mortalidade geral e por causas. Indicadores Sociais. Atlas de Econômico. $4^{\mathrm{a}}$ ed. [atualizado em 15 de fevereiro de 2019]. [citado 2019 Jul 10]. Disponível em: https://atlassocioeconomico.rs.gov.br/ coeficiente-de-mortalidade-geral-e-por-causas

13. Vieira MS. Perfil geográfico e clínico de pacientes admitidos na UTI através da Central de Regulação de Internações Hospitalares. Comun Ciênc Saúde. 2012;22:201-10.

14. Obermeyer Z, Abujaber S, Makar M, Stoll S, Kayden SR, Wallis LA, et al.; Acute Care Development Consortium. Emergency care in 59 lowand middle-income countries: a systematic review. Bull World Health Organ. 2015;93(8):577-586G.

15. Bezerra FC, Almeida Ml, Nóbrega-Therrien SM. Estudos sobre envelhecimento no Brasil: revisão bibliográfica. Rev Bras Geriatr Gerontol. 2012;15(1):155-67.

16. Tiné RF, Freitas CE, Paes NL. Paes, NL. Impact of the demographic transition on tax collection in Brazil: an analysis of the federative aspect. Estud Econ. 2020;50(1):43-65.

17. Cordeiro P, Martins M. Hospital mortality in older patients in the Brazilian Unified Health System, Southeast region. Rev Saude Publica. 2018:52:69

18. Machado JP, Martins M, Leite IC. Fontes de pagamento das internações e desempenho clínico: o caso dos hospitais do estado de São Paulo, Brasil. Saúde Debate. 2016;40(111):74-86.

19. Adhikari NK, Fowler RA, Bhagwanjee S, Rubenfeld GD. Critical care and the global burden of critical illness in adults. Lancet. 2010;376(9749):1339-46. 
20. Brasil. Ministério da Saúde. Saúde Brasil 2013 - uma análise da situação de saúde e das doenças transmissíveis relacionadas à pobreza. Brasília (DF): Ministério da Saúde; 2014. [citado 2019 Nov 10]. Disponível em: http://bvsms.saude.gov.br/bvs/publicacoes/ saude_brasil_2013_analise_situacao_saude.pdf

21. Izaias EM, Dellarosa MSG, Rossaneis MA, Belei RA. Custo e caracterização de infecção hospitalar em idosos. Cien Saúde Colet. 2014;19:3395-402.

22. Araújo PL, Mendonça AE, Souza-Neto VL, Medeiros RA, Nobre TT, Costa IK. Prevalência de infecção relacionada à assistência à saúde em pacientes internados em unidade de terapia intensiva. Enferm Global. 2018;52:291-303.

23. Souza IS, Santana AC, D’Alfonso Júnior G. A ocorrência de infecção do sítio cirúrgico: um estudo de revisão. Rev Med Minas Gerais. 2018:28(Supl 5):e-S280521.

24. Souza ES, Belei RA, Carrilho CM, Matsuo T, Yamada-Ogatta SF, Andrade $\mathrm{G}$, et al. Mortality and risks related to healthcare-associated infection. Texto Contexto Enferm. 2015;24(1):220-8.
25. Marra AR, Camargo LF, Pignatari AC, Sukiennik T, Behar PR, Medeiros EA, et al.; Brazilian SCOPE Study Group. Nosocomial bloodstream infections in Brazilian hospitals: analysis of 2,563 cases from a prospective nationwide surveillance study. J Clin Microbiol. 2011;49(5):1866-71.

26. Pagamisse AF, Tanner JJ, Poveda VB. Post-discharge surveillance of surgical site infections in teaching hospitals in Brazil. Rev Esc Enferm. USP 2020; 54:e03542.

27. Deptuła A, Trejnowska E, Ozorowski T, Hryniewicz W. Risk factors for healthcare-associated infection in light of two years of experience with the ECDC point prevalence survey of healthcare-associated infection and antimicrobial use in Poland. J Hosp Infect. 2015;90(4):310-5.

28. Khan HA, Baig FK, Mehboob R. Nosocomial infections: Epidemiology, prevention, control and surveillance. Asian Pac J Trop Biomed. 2017;7(5):478-82.

29. Watanabe ÉM, Almeida VF, Ottunes AF, Dessunti EM, Pieri FM, Martins EA, et al. Impacto das infecções relacionadas à assistência à saúde em pacientes acometidos por trauma. Semina: Ciênc Biológ Saúde. 2015;36(1 Supl):89-98. 